\title{
Perioperative Management of Elderly Patients with Gastrointestinal Malignancies: The Contribution of Anesthesia
}

\author{
Rudolf Mörgeli Kathrin Scholtz Johannes Kurth Sascha Treskatsch Bruno Neuner \\ Susanne Koch Lutz Kaufner Claudia Spies
}

Department of Anesthesiology and Operative Intensive Care Medicine (CCM, CVK), Charité Universitätsmedizin Berlin, corporate member of Freie Universität Berlin, Humboldt-Universität zu Berlin, and Berlin Institute of Health, Berlin, Germany

Keywords

Anesthesia · Perioperative · Elderly · Gastrointestinal · Malignancy

\section{Summary}

Introduction: Elderly patients suffering from gastrointestinal malignancies are particularly prone to perioperative complications. Elderly patients often present with reduced physiological reserves, and comorbidities can limit treatment options and promote complications. Surgeons and anesthesiologists must be aware of strategies required to deal with this vulnerable subgroup. Methods: We provide a brief review of current and emerging perioperative strategies for the treatment of elderly patients with gastrointestinal malignancies and frequent comorbidities. Results: Especially in combination with advanced age, the effects of malignancies can be devastating, bringing new health challenges, exacerbating preexisting conditions, and exerting severe psychological strain. An interdisciplinary assessment and process planning provide an ideal setting to identify and prevent potential complications, especially in regards to frailty and cardiovascular risk. In addition, important perioperative considerations are presented, such as malnutrition, fasting, intraoperative neuromonitoring, and hemodynamic control, as well as postoperative early mobilization, pain, and delirium management. Conclusion: The decisions and interventions made in the perioperative stage can positively influence many intraand postoperative factors, significantly improving the chances of successful treatment of elderly cancer patients. Appropriate management can help prevent or mitigate complications, secure a quick recovery, and improve short- and long-term outcomes.

(C) 2017 S. Karger GmbH, Freiburg

\section{Introduction}

There are several challenges associated with the provision of care to patients suffering from gastrointestinal malignancies, particularly in the elderly. Several studies, such as ERAS [1] and PERATECS [2], have already shown that the field of anesthesiology plays a significant role in ensuring the successful treatment and recovery of these patients. Surgeons and anesthesiologists must be aware of strategies required to deal with this special subgroup.

Regardless of age, malignancies usually entail massive physiological disturbances, such as anemia, muscle wasting, and organ dysfunctions, in addition to psychological strains like stress and anxiety. Therapeutic measures, such as chemotherapy, can be an additional burden on the body and are often associated with severe side effects.

In combination with advanced age, the effects of malignancies can be even more devastating. Elderly patients often already have reduced physiological reserves, and comorbidities can limit treatment options and promote complications.

Ultimately, special attention is required when treating this particularly vulnerable population. It is imperative to have an interdisciplinary, shared decision process when planning treatment so that their particular risk factors can be assessed and efficiently addressed. This article addresses a number of strategies that can be employed to improve the short- and long-term outcomes of geriatric oncological patients.

\section{Patient-Centered Outcome - Shared Decision Making}

The ultimate goal is not simply survival. All health care providers have the responsibility to administer care in such a way as to safeguard the patient's physical, psychosocial and cognitive integrity, both throughout the hospitalization period and after hospital discharge.

\section{KARGER \\ Fax +497614520714

() 2017 S. Karger GmbH, Freiburg 


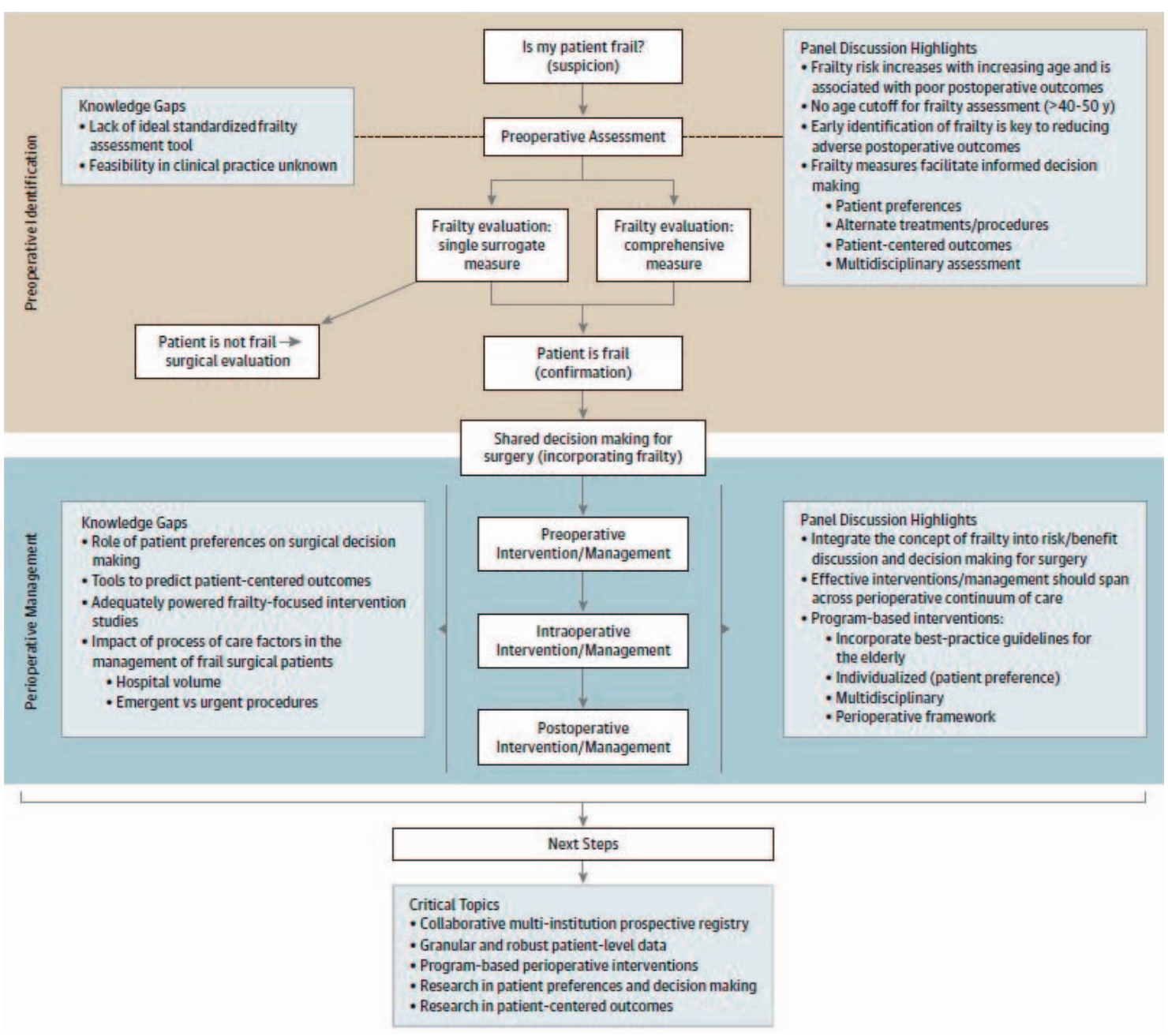

Fig. 1. Recommended evaluation and treatment processes for frail elders. Adapted from [3]. Reprinted with kind permission. (O) 2014 American Medical Association. All rights reserved.

In order to provide a patient-centered care, an interdisciplinary shared decision making process should take place for elderly oncological patients, with a focus on the patient's wishes and expectations. This team should consist of physicians from the surgical, anesthesiological, and geriatric specialties, as well as nurses, nutritionists, physiotherapists, and social workers [3]. It is important for patients to actively participate in this process; thus, it is crucial that they understand their situation, options, and risks. With the patient's consent, social networks should be activated and involved in the decision and treatment processes. An interdisciplinary team should analyze the individual risk factors of the patient, using all available information to reach an informed decision regarding any preoperative interventions, clearly define the extent and type of surgery and anesthesia required, and coordinate postoperative management, such as rehabilitation, analgesia, and nutritional support (fig. 1).

Especially in the context of frailty and cardiovascular risk factors, already established physical or cognitive impairments must be assessed, as these conditions are particularly prone to causing perioperative complications. Identification and treatment of these conditions can help improve the status of the patient before the operation $[4,5]$.

\section{Individual Risk Evaluation}

In order to identify emerging or undiagnosed conditions, the status of the elderly patient must be carefully and thoroughly scrutinized. Already diagnosed conditions should be carefully reassessed, ensuring that current therapies are adequate and effective.

Risk factors associated with gastrointestinal malignancies, such as substance abuse, diabetes, and inflammatory intestinal conditions, can cause severe perioperative complications and must be diagnosed (and treated) before surgery. Abstinence from nicotine and alcohol at least 1 month prior to the operation has been shown to reduce complication rates $[6,7]$.

Nutritional status is profoundly affected by most disease states, usually by a combination of increasing demand at a time when there is reduced intake. It is strongly recommended to carry out nutritional screening on all patients who are about to undergo major surgery [8]. The European Society for Clinical Nutrition and Metabolism (ESPEN) recommends using validated nutritional screening tools [9], such as the Nutrition Risk Screening (NRS2002), Mini Nutritional Assessment (MNA), and the Malnutrition Universal Screening Tool (MUST). A complete nutritional assessment should be carried out by positive screening results, including information on body mass index, food consumption, weight loss or 
Table 1. Severe nutritional risk markers, based on ESPEN Guidelines (modified from [88])

\section{Current body mass index $<18.5 \mathrm{~kg} / \mathrm{m}^{2}$}

Weight loss $>10-15 \%$ within the last 6 months

Serum albumin $<30 \mathrm{~g} / \mathrm{l}$ (with no evidence of hepatic/renal dysfunction)

$<80 \%$ of ideal body weight

gain, functional capacity, status of muscle mass and of subcutaneous fatty tissue, as well as localized or generalized fluid accumulation (which may sometimes mask weight loss). Evidence to support preoperative nutritional interventions is limited but suggests that the outcome is improved if malnourished individuals are adequately fed for at least 7-10 days before surgery [10]. Preoperative parenteral nutrition is indicated in case of severely malnourished patients (table 1) who cannot be adequately fed orally or enterally [11].

Laboratory tests should be performed to assess liver and kidney function, as an impaired metabolic function can require adjustments regarding the choice and dosage of anesthetics. Special attention should be paid to chemotherapy patients.

Even a mild anemia can lead to increased complication rates and mortality so that a preoperative anemia should be assessed and managed as early as possible $[12,13]$. For elective surgery, the hemoglobin level should be within the normal range of $12 \mathrm{~g} /$ $\mathrm{dl}_{(\mathrm{female})}$ and $13 \mathrm{~g} / \mathrm{dl}_{(\text {male) }}$, according to the NATA guidelines [14]. Also assess for clotting disorders, and always consider a planned epidural analgesia when preparing a thromboembolic prophylaxis protocol. The indication for blood products should also be anticipated and applied according to the transfusion guidelines. It is very important to consider preoperative anemia treatment instead of giving transfusions, which are known to increase the postoperative risk severalfold $[15,16]$.

Many elderly patients are also subject to polypharmacy ( $>5$ drugs), which should be prevented whenever possible by optimizing medication, both by giving preference to combination drugs and by adjusting dosages [17]. This is particularly relevant in avoiding long-term cognitive sequelae [18].

A cognitive assessment can help establish a preoperative cognitive deficit, which is prevalent in elderly patients, is rarely diagnosed, and known to be related to a steady and permanent postoperative long-term cognitive decline [19-21].

The implementation of a Comprehensive Geriatric Assessment for elderly cancer patients has been recommended by the International Society of Geriatric Oncology, as it enables the identification of thus far undiagnosed functional, psychological, social and pharmacological issues within this population [22]. Underlining the immense value of these measures, their utilization has been shown to reduce health care costs, readmission, and institutionalization rates [23, 24].

\section{Frailty Syndrome}

Frailty is being increasingly recognized as a major risk factor on the short- and long-term outcomes of elderly patients. The syndrome is multifactorial and frequently includes different, mainly physical, cognitive and psychosocial dimensions [25]. It refers to a reduced compensatory mechanism and lower reserves against stressors, rendering these individuals as particularly susceptible to intra- and postoperative complications, extended hospitalization, institutionalization, and mortality [26, 27].

While the individual factors making up the syndrome can usually be influenced, or even reversed, their combination is devastating. The physical aspects still dominate frailty assessment and diagnosis. Although there is no diagnostic gold standard, the most widely used definition is the Fried Physical Phenotype, which assesses physical activity, nutrition, mobility, energy, and muscle strength [28]. The cognitive aspects include memory, dementia, and cognitive impairments, while the psychosocial elements include, among others, depression, anxiety, social binding, living, and financial situation.

Malignancies can trigger or exacerbate several frailty factors (e.g. malnutrition, sarcopenia, depression) and thus cause a previously robust patient to become frail. Approximately $10 \%$ of all individuals over 65 years of age, however, are already frail [29], and the prevalence increases with age, so that more than half of all surgical procedures are ultimately performed on frail patients [30]. Awareness regarding this syndrome and the implementation of appropriate interventions are crucial for improving the survival and outcome of these patients [31]. It is suggested in this patient group to implement in-hospital and out-hospital shared decision making to empower patients and their relatives to participate in this obligatory interdisciplinary, multiprofessional process [3].

\section{Cardiovascular Risk}

Although the current guideline for preoperative evaluation of the European Society of Anesthesia (ESA) recommends focusing on the patient's risk factors rather than on age or frailty status, the number of cardiac comorbidities increases with age [32]. For non-cardiac surgery, the risk for a perioperative major cardiac event in an unselected population is estimated to be $1.4 \%$ [33], and this rate increases to $3.9 \%$ for patients with cardiac risk factors [34]. In addition, the classification system of the European Society of Cardiology (ESC), which assesses the risk of a major cardiac event within 30 days of surgery, ranks intraperitoneal, duodeno-pancreatic, liver and perforated bowel surgery as intermediate- to high-risk surgical procedures [33]. In order to improve outcome, surgery in patients at risk should include advanced hemodynamic monitoring as well as an individualized goal-directed therapy (GDT) throughout the procedure.

Cardiac risk varies widely between procedures, depending on factors such as duration of procedure, blood loss, fluid shifts, anatomical region, and patients' positioning. Tissue damage caused by surgery leads to an inflammatory response and a neuroendocrinemediated sympathovagal imbalance, which increases systemic and especially myocardial oxygen demand. Therefore, fluid imbalance can increase surgical stress and oxygen demand while further reducing oxygen supply [35].

Thus, when assessing a patient's medical history, it is crucial to focus on functional capacity as well as on symptoms of diastolic and systolic heart failure. 
A preoperative evaluation of functional capacity is also needed to estimate the patient's ability for coping with surgical stress, as functional impairment has been linked to higher 1-year mortality in elderly gastrointestinal cancer patients [36]. Metabolic equivalents (MET) are used to quantify functional capacity, whereas 1 MET reflects the metabolic rate at rest and $>10$ METs depict the metabolic rate during exertive sports. A poor functional capacity is reflected by the inability to climb two flights of stairs or to run a short distance, and a MET $<4$ is associated with an increased incidence of cardiac events following non-cardiac surgery [35, 37].

The Revised Cardiac Risk Index (RCRI) is a relatively simple method used for perioperative risk stratification, focusing on arteriosclerosis-related causes and comorbidities [33]. In patients with an RCRI $\geq 2$ and poor functional capacity, non-invasive imaging stress testing is recommended before high-risk surgery, while preoperative elective angiography is recommended in patients with proven myocardial ischemia under medical therapy and unstable angina pectoris [35].

In addition, biomarkers, such as cardiac troponins or brain-related natriuretic peptides, may be considered in patients with impaired functional capacity (MET $\leq 4)$ or with an RCRI $\geq 1$ for vascular surgery and $\geq 2$ for non-vascular surgery [35].

Finally, transthoracic echocardiography (TTE) is a non-invasive, versatile, readily available diagnostic tool that can provide both structural and functional information. TTE can assess left and right ventricular systolic, diastolic (such as E/e' ratio), and valve function to significantly predict cardiac complications, and is also recommended in asymptomatic patients before high surgical risk [35]. If myocardial/ventricular heart disease or congestive heart failure is known or suspected, an echocardiographic evaluation should take place in all elective intermediate or high-risk non-cardiac surgery [35]. Preoperative echocardiography should be also be considered early in frail patients. Frailty is linked to a decreased left ventricular function, a higher left ventricular mass index, a higher left atrial volume index, an impaired myocardial relaxation, and an increased pulmonary artery pressure [38]. In addition, a preoperative TTE at rest may help in guiding intraoperative hemodynamic management.

\section{Preoperative Management}

The decisions and interventions made at the beginning of the perioperative stage can positively influence many intra- and postoperative factors, significantly improving the chances of successful treatment of elderly cancer patients. Fasting, premedication, fluid management, and antibiotics are some of the aspects that must be considered with diligence and caution.

\section{Preparation for Surgery}

Preoperative fasting should be restricted to $6 \mathrm{~h}$ without solid food and $2 \mathrm{~h}$ without clear fluids prior to induction [39]. In addition, preoperative fluid restrictions were the most predictive factor for postoperative delirium (POD) in the recovery room [40]. Due to a reduced sense of taste and thirst, dehydration and malnutrition are often seen in elderly patients, and gastrointestinal malignancies can exacerbate this picture with nausea, chemotherapeutic side effects, or a prolonged gastric clearance. Especially for operations scheduled for the early morning, fasting periods can be significantly longer. Diabetes and cardiovascular conditions can be easily aggravated by prolonged fasting, and thus, it is important to encourage the patient to strictly adhere to the prescribed period as well as to avoid or timely communicate any scheduling delays. The administration of intravenous fluids should be considered for elderly patients at risk as well as due to significant surgery delays. Small studies have shown that preoperative carbohydrate loading on the previous evening and $2-3 \mathrm{~h}$ before the operation can improve outcome [41, 42].

Premedication with benzodiazepines should be avoided whenever possible, as their use has been associated with a higher incidence of postoperative complications such as delirium [43]. It is important, however, not to deny premedication to patients with severe anxiety, as untreated patients are also at higher risk for complications [44]. In such cases, dosage adjustments should be considered. Beware of adverse reactions, such as respiratory suppression, and ensure that premedicated patients are continuously monitored. Premedication, as well as age, can alter the reference levels in electroencephalography (EEG), so EEG monitoring should be started prior to induction of anesthesia in all patients [45].

In order to reduce the risk of postoperative infections, antibiotics should be administered between $30 \mathrm{~min}$ and $1 \mathrm{~h}$ before surgery [46], either at the ward or immediately upon arrival in the operating room. In addition, thermoregulation can also be impaired in elderly patients, and they tend to have a limited compensatory mechanism due to reduced muscle mass or impaired circulation. They should be promptly covered and actively warmed upon arrival, even before placement of a peridural catheter, as even mild hypothermia has been shown to disrupt clotting and increase postoperative infection rates [47].

\section{Induction of Elderly Patients}

Anesthesia induction of elderly patients must take their current physical state and comorbidities into consideration.

While regional anesthesia is not feasible for gastrointestinal surgery, a patient-controlled epidural analgesia system can be used to reduce the amount of opioids required during and after the operation. The systems also allow for faster mobilization, a greater level of independence from staff during the postoperative period, and intestinal stimulation through inhibition of the sympathetic innervation $[1,48]$.

Reduced proportions of muscle and fat, as well as a reduced neural mass, dehydration, and organ dysfunctions, can alter drug distribution and metabolism considerably [49]. This change in drug pharmacodynamics and pharmacokinetics means that routine dosages can have toxic effects in the elderly oncologic patient. This altered state must be anticipated, and the choice and dosage of substances should be adapted to avoid subsequent complications. Ideally, induction and maintenance of anesthesia should be performed 
with short-acting substances, preferably independent of renal or hepatic elimination [50].

When dealing with gastrointestinal tumors, a rapid sequence induction should be considered for patients with nausea, delayed gastric passage, or subileus symptomatology. Prophylaxis for postoperative nausea and vomiting should be given during or immediately after induction.

High-risk patients should have 12-lead electrocardiography monitoring throughout the surgical procedure in order to improve sensitivity for the detection of myocardial ischemia [35].

\section{Intraoperative Management}

There are a number of intraoperative considerations and precautions that must be implemented during anesthesia of this vulnerable patient collective. In particular, neuro- and cardiovascular monitoring can improve patient safety during medium- and highrisk operations, allowing for the rapid assessment and treatment of potential complications.

\section{General Considerations}

Special care is required for the positioning of weakened patients for surgery, particularly for long procedures. Brittle skin, poor circulation, and reduced fat and muscle mass make these patients prone to neural damage, decubitus, and thrombosis. Positioning should be regularly controlled in order to detect unwanted shifting during the operation.

Extreme blood pressure fluctuations should be avoided whenever possible, as variation is not as easily tolerated in the frail or severely ill [51]. These changes can occur in response to medication, fluid shift, blood loss, intraoperative positioning, or surgical stimuli.

Therapy responses must be regularly controlled, including an appropriate urinary output, blood gas analyses for blood loss or fluid/blood product application, temperature measurement by active warming, as well as depth and duration of a neuromuscular blockade by relaxant administration (especially before emergence from anesthesia).

\section{Neuromonitoring}

To aid in the titration of anesthetics, neuromonitoring should be routinely employed in elderly patients. Although the use of EEG during surgery is widely recommended, it is still poorly implemented. EEG evaluations can be challenging, and tools have been developed to help interpret readings, such as the use of spectrograms and index values. Several studies are dealing with intraoperative EEG dynamics and evaluation, paving the way to newer and more effective strategies. Such studies have recently shown a reduced alpha band power in elderly patients during unconsciousness as well as an elevated risk to develop burst suppression state during anesthesia [52]. This is crucial since avoidance of burst suppression states (fig. 2) during anesthesia can help in safeguarding the patients from debilitation and sometimes permanent, post-

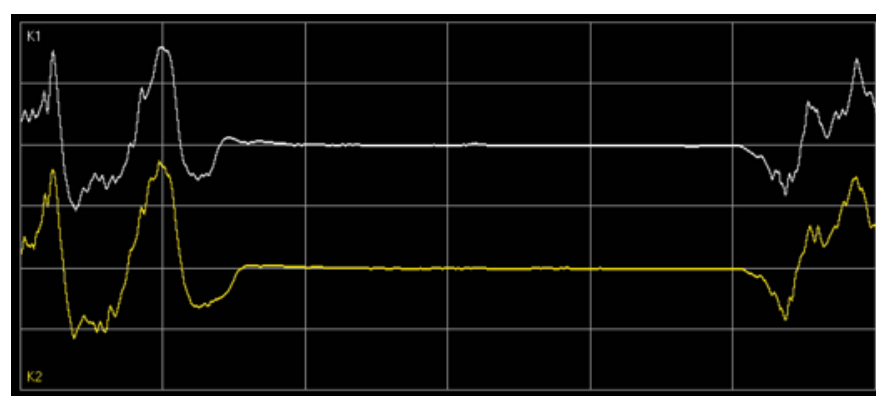

Fig. 2. Burst suppression state in electroencephalogram, recognizable as an intermittent and synchronous 'flatline' of all brain waves.

operative cognitive complications [53, 54]. For now, monitoring the substance-specific EEG dynamic of a frontal alpha band coherence, related to a GABA-induced unconscious state, can help to ensure an appropriate level of anesthesia $[55,56]$. In elderly patients with reduced alpha band power, monitoring might be facilitated by increasing the power scale from 60 to 30 or $15 \mu \mathrm{V}$, so as to better delineate the alpha band (feasible in most monitoring systems).

\section{Hemodynamics and Goal-Directed Fluid Therapy}

An individualized hemodynamic optimization is essential in the perioperative course. The concept of GDT was described for the treatment of high-risk surgical patients as early as 1993 [57], aiming to improve tissue perfusion and oxygen delivery (DO2) via an optimized application of fluids, red blood cells, inotropic and/or vasoconstrictive therapy, as well as oxygen [58]. In this context, cardiac output is the major determinant for oxygen delivery [59]. In the perioperative course, GDT has been shown to decrease mortality, postoperative complications, morbidity, and length of stay on the intensive care unit (ICU) $[60,61]$. Surgical stress and inflammatory responses lead to increased oxygen consumption, while oxygen supply is decreased by blood loss and fluid shifts. This divergence often initiates a vicious circle, as attempts to increase cardiac output lead to an increased oxygen consumption and shortened diastolic filling, subsequently lowering oxygen delivery for the heart itself. A decreased preload due to excessive fluid restriction leads to hypovolemia and an increased rate of postoperative complications [60]. Conversely, a volume overload can lead to congestion and heart failure, also prolonging hospital length of stay [62].

Several GDT strategies and hemodynamic monitoring devices have been introduced into clinical practice [61, 63]. However, atrial fibrillation, right ventricular dysfunction, and valve regurgitation may reduce their validity [64-66]. In this context, transesophageal echocardiography (TEE) is recently becoming more important as it may influence volume and catecholamine therapies [67]. Compared to TTE, TEE also provides structural, functional and hemodynamic information, especially when a transthoracic approach is not feasible intraoperatively. TEE is already recommended in highrisk surgery and should always be used if unexplained life-threatening hypotension persists despite corrective therapy, as well as in 
unexplained hypoxemia [38]. Since perioperative TEE is usually performed by anesthesiologists in high-stress situations, straightforward protocols for the examination and a special training of the examiner are indispensable [68].

\section{Postoperative Management}

Avoiding postoperative complications is critical for a speedy recovery. Many potential complications can be treated or mitigated if diagnosis is made early, so vigilance is required. Depending on the status of the patient and the extent of the operation, an ICU stay may be required [69]. An appropriate delirium and pain management as well as a continuing nutritional support and an early mobilization can help ensure that the length of hospitalization remains as short as possible. Social binding becomes critical at this stage, as support from family and friends can not only guard against depression and anxiety but also enable an early hospital discharge.

\section{Delirium and Pain Management}

There are important guidelines regarding patient management in regards to POD [44]. POD is an acute cerebral dysfunction and a common complication affecting about a quarter of elderly patients following major abdominal surgery [70]. The condition has been shown to increase mortality, and $55 \%$ of delirious patients go on to develop long-lasting cognitive deficits [71]. When recognized, POD is treatable, and its ramifications can be limited or avoided if a rapid symptomatic treatment is initiated. Since POD is often fluctuating, patient assessment must take place regularly by using validated tools not only on the ICU or in the recovery room but also in the peripheral wards. Validated tools include the Nursing Delirium Screening Scale [72] and the Confusion Assessment Method [73].

Pain management should also include regular assessments using validated tools. In order to reduce perioperative stress and to avoid complications, choice and dose of analgesics must be carefully selected. A patient-controlled analgesia has been shown to improve mobility, reduce stress, and better titrate the overall analgesic intake of the patients. The use of opioids can be problematic, as side effects, e.g. nausea and obstipation, can be particularly strenuous following gastrointestinal surgery. Therefore, the goal is to reduce opioid requirements and to limit their side effects as well as to keep patients alert and oriented, thus enabling them to actively participate in their recovery.

\section{Nutritional Support}

Nutritional support should be continued after surgery. An early resumption of postoperative nutrition has been shown to benefit the patients [74]. Early oral feeding after gastric oncological surgery seems feasible and safe, and should be started at the day of surgery, irrespective of the extent of the gastric resection and the type of surgery [75]. This also applies to early nutrition following colorectal resections, as results show that this practice is safe and feasible [76].
All patients who are not expected to be on a full oral diet within 3 days should receive enteral nutrition [77]. Experimental data demonstrate the benefit of the early initiation of enteral nutrition [78]. It has since been shown that postoperative enteral feeding in patients undergoing gastrointestinal resection is safe and well tolerated even when started within $12 \mathrm{~h}$ of surgery [79]. In patients who require postoperative artificial nutrition, enteral feeding or a combination of enteral and supplementary parenteral feeding is the first choice [79].

There is also evidence that enteral feeding initiated immediately after major colorectal surgery, in patients given preoperative carbohydrates and thoracic epidural anesthesia, normalized glucose levels and was associated with a suppression of the catabolic response to surgery [80]. These findings suggest that it is possible to overcome most of the metabolic response to injury with a combination of postoperative feeding, preoperative carbohydrates, and epidural anesthesia.

Postoperative parenteral nutrition is beneficial for undernourished patients in whom enteral nutrition is not feasible or not tolerated, as well as in patients with postoperative complications impairing gastrointestinal function who are unable to receive and absorb adequate amounts of oral/enteral feeding for at least 7 days [11].

\section{Early Mobilization}

There is sufficient evidence that patients in poor physical condition and with a low anaerobic threshold have greater postoperative morbidity and mortality rates [81]. Mobilization during the first 24 $\mathrm{h}$ after surgery is recommended and has been successfully employed in the ERAS program, whose patients were shown to achieve independent mobilization sooner [82]. Early and frequent ambulation and alimentation is associated with decreased complications and readmissions in adult patients admitted for elective small or large bowel operations [83], and loss of muscle mass and function can be attenuated by early mechanical loading and early physical therapy in immobilized patients [84-86].

Patients mobilized during the first days in an ICU (even under mechanical ventilation) do not only have a better muscular activity but also an improved vascular and respiratory function as well as a reduced risk of delirium [87].

\section{Complications}

As a high-risk group, it is important to anticipate and monitor several possible postoperative complications, such as anastomosis insufficiency, kidney failure, and infections. An appropriate decubitus and thrombosis prophylaxis protocol should be implemented.

Potential infection sites, e.g. central lines and catheters, should be regularly inspected. Fever and infection parameters should be monitored frequently, and pain should be taken seriously. The use of a peridural catheter can temporarily mask surgical complications like anastomosis insufficiency; therefore, a sudden increase in analgesic requirements should be critically evaluated. 


\section{Conclusions}

The elderly patient suffering from a gastrointestinal malignancy is particularly prone to perioperative complications. An interdisciplinary assessment and process planning provide an ideal setting to identify and prevent potential issues, especially in regards to frailty and cardiovascular risk factors.

Several anesthesiological strategies for the pre-, intra- and postoperative management of such patients can help prevent or miti- gate complications, securing a quick recovery and improving short- and long-term outcomes.

\section{Disclosure Statement}

The authors have no conflicts of interest to disclose with respect to this manuscript; JCMJE COI forms are available to the journal editors.

\section{References}

1 Feldheiser A, Aziz O, Baldini G, et al: Enhanced Recovery After Surgery (ERAS) for gastrointestinal surgery, part 2: consensus statement for anaesthesia practice. Acta Anaesthesiol Scand 2016;60:289-334.

2 Schmidt M, Eckardt R, Scholtz K, Neuner B, von Dossow-Hanfstingl V, Sehouli J, Stief CG, Wernecke K-D, Spies CD; PERATECS Group: Patient empowerment improved perioperative quality of care in cancer patients aged $\geq 65$ years - a randomized controlled trial. PLoS One 2015;10:e0137824.

3 Anaya DA, Johanning J, Spector SA, Katlic MR, Perrino AC, Feinleib J, Rosenthal RA: Summary of the panel session at the 38th Annual Surgical Symposium of the Association of VA Surgeons: what is the big deal about frailty? JAMA Surg 2014;149:1191-1197.

4 de Vries NM, van Ravensberg CD, Hobbelen JSM, Olde Rikkert MGM, Staal JB, Nijhuis-van der Sanden MWG: Effects of physical exercise therapy on mobility, physical functioning, physical activity and quality of life in community-dwelling older adults with impaired mobility, physical disability and/or multi-morbidity: a meta-analysis. Ageing Res Rev 2012;11:136-149.

5 Crocker T, Forster A, Young J, et al: Physical rehabilitation for older people in long-term care. Cochrane Database Syst Rev 2013;(2):CD004294.

6 Wong J, Lam DP, Abrishami A, Chan MTV, Chung F: Short-term preoperative smoking cessation and postoperative complications: a systematic review and metaanalysis. Can J Anaesth 2012;59:268-279.

7 Tønnesen H, Nielsen PR, Lauritzen JB, Møller AM: Smoking and alcohol intervention before surgery: evidence for best practice. Br J Anaesth 2009;102:297306.

8 Cerantola Y, Grass F, Cristaudi A, Demartines N, Schäfer M, Hübner M: Perioperative nutrition in abdominal surgery: recommendations and reality. Gastroenterol Res Pract 2011;2011:739347.

9 Kondrup J, Allison SP, Elia M, Vellas B, Plauth M; Educational and Clinical Practice Committee; European Society of Parenteral and Enteral Nutrition (ESPEN): ESPEN guidelines for nutrition screening 2002. Clin Nutr 2003;22:415-421.

10 Ward N: Nutrition support to patients undergoing gastrointestinal surgery. Nutr J 2003;2:18.

11 Braga M, Ljungqvist O, Soeters $\mathrm{P}$, Fearon K, Weimann A, Bozzetti F; ESPEN: ESPEN Guidelines on Parenteral Nutrition: surgery. Clin Nutr 2009;28:378-386.

12 Musallam KM, Tamim HM, Richards T, et al: Preoperative anaemia and postoperative outcomes in noncardiac surgery: a retrospective cohort study. Lancet 2011;378:1396-1407.
13 Gupta PK, Sundaram A, Mactaggart JN, et al: Preoperative anemia is an independent predictor of postoperative mortality and adverse cardiac events in elderly patients undergoing elective vascular operations. Ann Surg 2013;258:1096-1102.

14 Goodnough LT, Maniatis A, Earnshaw P, et al: Detection, evaluation, and management of preoperative anaemia in the elective orthopaedic surgical patient: NATA guidelines. Br J Anaesth 2011;106:13-22.

15 von Heymann C, Kaufner L, Sander M, Spies C, Schmidt K, Gombotz H, Wernecke K-D, Balzer F: Does the severity of preoperative anemia or blood transfusion have a stronger impact on long-term survival after cardiac surgery? J Thorac Cardiovasc Surg 2016;152:1412-1420.

16 Muñoz M, Acheson AG, Auerbach M, et al: International consensus statement on the peri-operative management of anaemia and iron deficiency. Anaesthesia 2017;72:233-247.

17 Ancelin ML, Artero S, Portet F, Dupuy A-M, Touchon J, Ritchie K: Non-degenerative mild cognitive impairment in elderly people and use of anticholinergic drugs: longitudinal cohort study. BMJ 2006;332:455-459.

18 Carnahan RM, Lund BC, Perry PJ, Pollock BG, Culp KR: The Anticholinergic Drug Scale as a measure of drug-related anticholinergic burden: associations with serum anticholinergic activity. J Clin Pharmacol 2006; 46:1481-1486.

19 Plassman BL, Langa KM, Fisher GG, et al: Prevalence of cognitive impairment without dementia in the United States. Ann Intern Med 2008;148:427-434.

20 Feinkohl I, Winterer G, Spies CD, Pischon T: Cognitive reserve and the risk of postoperative cognitive dysfunction. Dtsch Arzteblatt Int 2017;114:110-117.

21 Steinmetz J, Christensen KB, Lund T, Lohse N, Rasmussen LS; ISPOCD Group: Long-term consequences of postoperative cognitive dysfunction. Anesthesiology 2009;110:548-555.

22 Extermann M, Aapro M, Bernabei R, et al: Use of comprehensive geriatric assessment in older cancer patients: recommendations from the task force on CGA of the International Society of Geriatric Oncology (SIOG). Crit Rev Oncol Hematol 2005;55:241-252.

23 Flood KL, Maclennan PA, McGrew D, Green D, Dodd C, Brown CJ: Effects of an acute care for elders unit on costs and 30-day readmissions. JAMA Intern Med 2013;173:981-987.

24 Landefeld CS, Palmer RM, Kresevic DM, Fortinsky $\mathrm{RH}$, Kowal J: A randomized trial of care in a hospital medical unit especially designed to improve the functional outcomes of acutely ill older patients. N Engl J Med 1995;332:1338-1344.
25 Inouye SK, Studenski S, Tinetti ME, Kuchel GA: Geriatric syndromes: clinical, research, and policy implications of a core geriatric concept. J Am Geriatr Soc 2007;55:780-791.

26 Farhat JS, Velanovich V, Falvo AJ, Horst HM, Swartz A, Patton JH, Rubinfeld IS: Are the frail destined to fail? Frailty index as predictor of surgical morbidity and mortality in the elderly. J Trauma Acute Care Surg 2012;72:1526-1530; discussion 1530-1531.

27 Lin H-S, Watts JN, Peel NM, Hubbard RE: Frailty and post-operative outcomes in older surgical patients: a systematic review. BMC Geriatr 2016;16:157.

28 Fried LP, Tangen CM, Walston J, et al: Frailty in older adults: evidence for a phenotype. J Gerontol A Biol Sci Med Sci 2001;56:M146-156.

29 Aarts S, Patel KV, Garcia ME, et al: Co-presence of multimorbidity and disability with frailty: an examination of heterogeneity in the frail older population. J Frailty Aging 2015;4:131-138.

30 Etzioni DA, Liu JH, Maggard MA, Ko CY: The aging population and its impact on the surgery workforce. Ann Surg 2003;238:170-177.

31 Hall DE, Arya S, Schmid KK, Carlson MA, Lavedan P, Bailey TL, Purviance G, Bockman T, Lynch TG, Johanning JM: Association of a frailty screening initiative with postoperative survival at 30,180 , and 365 days. JAMA Surg 2016;152:233-240.

32 De Hert S, Imberger G, Carlisle J, et al: Preoperative evaluation of the adult patient undergoing non-cardiac surgery: guidelines from the European Society of Anaesthesiology. Eur J Anaesthesiol 2011;28:684-722.

33 Lee TH, Marcantonio ER, Mangione CM, et al: Derivation and prospective validation of a simple index for prediction of cardiac risk of major noncardiac surgery. Circulation 1999;100:1043-1049.

34 Devereaux PJ, Goldman L, Cook DJ, Gilbert K, Leslie K, Guyatt GH: Perioperative cardiac events in patients undergoing noncardiac surgery: a review of the magnitude of the problem, the pathophysiology of the events and methods to estimate and communicate risk. CMAJ 2005; 173:627-634

35 Kristensen SD, Knuuti J, Saraste A, et al: 2014 ESC/ ESA Guidelines on non-cardiac surgery: cardiovascular assessment and management: The Joint Task Force on non-cardiac surgery: cardiovascular assessment and management of the European Society of Cardiology (ESC) and the European Society of Anaesthesiology (ESA). Eur J Anaesthesiol 2014;31:517-573.

36 Schmidt M, Eckardt R, Sehouli J, Wernecke KD, Spies C: Functional impairment prior to major non-cardiac surgery is associated with mortality within one year in elderly patients with gastrointestinal, gynaecological and urogenital cancer: a prospective observational cohort study. J Geriatr Oncol 2017;in press. 
37 Biccard BM: Relationship between the inability to climb two flights of stairs and outcome after major non-cardiac surgery: implications for the pre-operative assessment of functional capacity. Anaesthesia 2005; 60:588-593.

38 Leibowitz D, Jacobs JM, Stessman-Lande I, Cohen A, Gilon D, Ein-Mor E, Stessman J: Cardiac structure and function and dependency in the oldest old. J Am Geriatr Soc 2011;59:1429-1434.

39 Smith I, Kranke P, Murat I, Smith A, O'Sullivan G, Søreide E, Spies C, in’t Veld B; European Society of Anaesthesiology: Perioperative fasting in adults and children: guidelines from the European Society of Anaesthesiology. Eur J Anaesthesiol 2011;28:556-569.

40 Radtke FM, Franck M, MacGuill M, Seeling M, Lütz A, Westhoff S, Neumann U, Wernecke KD, Spies CD Duration of fluid fasting and choice of analgesic are modifiable factors for early postoperative delirium. Eur J Anaesthesiol 2010;27:411-416.

41 Hausel J, Nygren J, Thorell A, Lagerkranser M, Ljungqvist O: Randomized clinical trial of the effects of oral preoperative carbohydrates on postoperative nausea and vomiting after laparoscopic cholecystectomy. Br J Surg 2005;92:415-421.

42 Yagci G, Can MF, Ozturk E, Dag B, Ozgurtas T, Cosar A, Tufan T: Effects of preoperative carbohydrate loading on glucose metabolism and gastric contents in patients undergoing moderate surgery: a randomized, controlled trial. Nutrition 2008;24:212-216.

43 Radtke FM, Franck M, Hagemann L, Seeling M, Wernecke KD, Spies CD: Risk factors for inadequate emergence after anesthesia: emergence delirium and hypoactive emergence. Minerva Anestesiol 2010;76:394-403.

44 Aldecoa C, Bettelli G, Bilotta F, et al: European Society of Anaesthesiology evidence-based and consensusbased guideline on postoperative delirium. Eur J Anaesthesiol 2017;34:192-214.

45 Blume WT: Drug effects on EEG. J Clin Neurophysiol 2006;23:306-311.

46 Steinberg JP, Braun BI, Hellinger WC, et al: Timing of antimicrobial prophylaxis and the risk of surgical site infections: results from the Trial to Reduce Antimicrobial Prophylaxis Errors. Ann Surg 2009;250:10-16.

47 Putzu M, Casati A, Berti M, Pagliarini G, Fanelli G: Clinical complications, monitoring and management of perioperative mild hypothermia: anesthesiological features. Acta Biomed 2007;78:163-169.

48 Marret E, Remy C, Bonnet F; Postoperative Pain Forum Group: Meta-analysis of epidural analgesia versus parenteral opioid analgesia after colorectal surgery. Br J Surg 2007;94:665-673.

49 Grandison MK, Boudinot FD: Age-related changes in protein binding of drugs: implications for therapy. Clin Pharmacokinet 2000;38:271-290.

50 Rundshagen I: Anaesthesiological strategies for old patients (Article in German). Anästh Intensivmed 2015; 56:534-545.

51 Radtke FM, Franck M, Schust S, et al: A comparison of three scores to screen for delirium on the surgical ward. World J Surg 2010;34:487-494.

52 Purdon PL, Pavone KJ, Akeju O, Smith AC, Sampson AL, Lee J, Zhou DW, Solt K, Brown EN: The ageing brain: age-dependent changes in the electroencephalogram during propofol and sevoflurane general anaesthesia. Br J Anaesth 2015;115(suppl 1):i46-i57.

53 Radtke FM, Franck M, Lendner J, Krüger S, Wernecke $\mathrm{KD}$, Spies CD: Monitoring depth of anaesthesia in a randomized trial decreases the rate of postoperative delirium but not postoperative cognitive dysfunction. Br J Anaesth 2013;110(suppl 1):i98-105.
54 Whitlock EL, Torres BA, Lin N, Helsten DL, Nadelson MR, Mashour GA, Avidan MS: Postoperative delirium in a substudy of cardiothoracic surgical patients in the BAG-RECALL clinical trial. Anesth Analg 2014;118: 809-817.

55 Purdon PL, Pierce ET, Mukamel EA, et al: Electroencephalogram signatures of loss and recovery of consciousness from propofol. Proc Natl Acad Sci U S A 2013;110:E1142-1151.

56 Akeju O, Westover MB, Pavone KJ, Sampson AL, Hartnack KE, Brown EN, Purdon PL: Effects of sevoflurane and propofol on frontal electroencephalogram power and coherence. Anesthesiology 2014;121:990998.

57 Shoemaker WC, Appel PL, Kram HB: Hemodynamic and oxygen transport responses in survivors and nonsurvivors of high-risk surgery. Crit Care Med 1993;21: 977-990.

58 Della Rocca G, Pompei L: Goal-directed therapy in anesthesia: any clinical impact or just a fashion? Minerva Anestesiol 2011;77:545-553.

59 McGee WT, Raghunathan K: Physiologic goal-directed therapy in the perioperative period: the volume prescription for high-risk patients. J Cardiothorac Vasc Anesth 2013;27:1079-1086.

60 Futier E, Constantin J-M, Petit A, Chanques G, Kwiatkowski F, Flamein R, Slim K, Sapin V, Jaber S, Bazin J-E: Conservative vs restrictive individualized goal-directed fluid replacement strategy in major abdominal surgery: a prospective randomized trial. Arch Surg 2010;145:1193-1200.

61 Hamilton MA, Cecconi M, Rhodes A: A systematic review and meta-analysis on the use of preemptive hemodynamic intervention to improve postoperative outcomes in moderate and high-risk surgical patients. Anesth Analg 2011;112:1392-1402.

62 Thacker JK, Mountford WK, Ernst FR, Krukas MR, Mythen MM: Perioperative fluid utilization variability and association with outcomes: considerations for enhanced recovery efforts in sample US surgical populations. Ann Surg 2016;263:502-510.

63 Vincent J-L, Pelosi P, Pearse R, et al: Perioperative cardiovascular monitoring of high-risk patients: a consensus of 12. Crit Care 2015;19:224.

64 Ostergaard M, Nilsson LB, Nilsson JC, Rasmussen JP, Berthelsen PG: Precision of bolus thermodilution cardiac output measurements in patients with atrial fibrillation. Acta Anaesthesiol Scand 2005;49:366-372.

65 Ranucci M, Pazzaglia A, Tritapepe L, et al: Fluid responsiveness and right ventricular function in cardiac surgical patients. A multicenter study. HSR Proc Intensive Care Cardiovasc Anesth 2009;1:21-29.

66 Balik M, Pachl J, Hendl J : Effect of the degree of tricuspid regurgitation on cardiac output measurements by thermodilution. Intensive Care Med 2002;28:11171121.

67 Carl M, Alms A, Braun J, et al: S3 guidelines for intensive care in cardiac surgery patients: hemodynamic monitoring and cardiocirculary system. Ger Med Sci 2010;8:Doc12.

68 Treskatsch S, Balzer F, Knebel F, Habicher M, Braun JP, Kastrup M, Grubitzsch H, Wernecke K-D, Spies C, Sander M: Feasibility and influence of hTEE monitoring on postoperative management in cardiac surgery patients. Int J Cardiovasc Imaging 201531:1327-1335.

69 Pearse RM, Moreno RP, Bauer P, et al: Mortality after surgery in Europe: a 7 day cohort study. Lancet 2012; 380:1059-1065.

70 Morimoto Y, Yoshimura M, Utada K, Setoyama K, Matsumoto M, Sakabe T: Prediction of postoperative delirium after abdominal surgery in the elderly. J Anesth 2009;23:51-56.
71 Saczynski JS, Marcantonio ER, Quach L, Fong TG, Gross A, Inouye SK, Jones RN: Cognitive trajectories after postoperative delirium. N Engl J Med 2012;367: 30-39.

72 Gaudreau J-D, Gagnon P, Harel F, Tremblay A, Roy M-A: Fast, systematic, and continuous delirium assessment in hospitalized patients: the nursing delirium screening scale. J Pain Symptom Manage 2005;29: 368-375.

73 Inouye SK, van Dyck CH, Alessi CA, Balkin S, Siegal AP, Horwitz RI: Clarifying confusion: the confusion assessment method. A new method for detection of delirium. Ann Intern Med 1990;113:941-948.

74 Lewis SJ, Andersen HK, Thomas S: Early enteral nutrition within $24 \mathrm{~h}$ of intestinal surgery versus later commencement of feeding: a systematic review and metaanalysis. J Gastrointest Surg 2009;13:569-575.

75 Liu X, Wang D, Zheng L, Mou T, Liu H, Li G: Is early oral feeding after gastric cancer surgery feasible? A systematic review and meta-analysis of randomized controlled trials. PloS One 2014;9:e112062.

76 Fujii T, Morita H, Sutoh T, Yajima R, Yamaguchi S, Tsutsumi S, Asao T, Kuwano H: Benefit of oral feeding as early as one day after elective surgery for colorectal cancer: oral feeding on first versus second postoperative day. Int Surg 2014;99:211-215.

77 Kreymann KG, Berger MM, Deutz NEP, et al: ESPEN Guidelines on Enteral Nutrition: intensive care. Clin Nutr 2006;25:210-223.

78 Marik PE, Zaloga GP: Early enteral nutrition in acutely ill patients: a systematic review. Crit Care Med 2001; 29:2264-2270.

79 Braga M, Gianotti L, Gentilini O, Liotta S, Di Carlo V: Feeding the gut early after digestive surgery: results of a nine-year experience. Clin Nutr 200221:59-65.

80 Soop M, Carlson GL, Hopkinson J, Clarke S, Thorell A, Nygren J, Ljungqvist O: Randomized clinical trial of the effects of immediate enteral nutrition on metabolic responses to major colorectal surgery in an enhanced recovery protocol. Br J Surg 2004;91:1138-1145.

81 Wilson RJT, Davies S, Yates D, Redman J, Stone M Impaired functional capacity is associated with allcause mortality after major elective intra-abdominal surgery. Br J Anaesth 2010;105:297-303.

82 Lassen K, Soop M, Nygren J, et al: Consensus review of optimal perioperative care in colorectal surgery: Enhanced Recovery After Surgery (ERAS) Group recommendations. Arch Surg 2009;144:961-969.

83 Loftus TJ, Stelton S, Efaw BW, Bloomstone J: A system-wide enhanced recovery program focusing on two key process steps reduces complications and readmissions in patients undergoing bowel surgery. J Healthc Qual 2017;39:129-135.

84 Llano-Diez M, Renaud G, Andersson M, Marrero HG Cacciani N, Engquist H, Corpeño R, Artemenko K, Bergquist J, Larsson L: Mechanisms underlying ICU muscle wasting and effects of passive mechanical loading. Crit Care 2012;16:R209.

85 Bailey P, Thomsen GE, Spuhler VJ, Blair R, Jewkes J Bezdjian L, Veale K, Rodriquez L, Hopkins RO: Early activity is feasible and safe in respiratory failure patients. Crit Care Med 2007;35:139-145.

86 Kayambu G, Boots R, Paratz J: Physical therapy for the critically ill in the ICU: a systematic review and metaanalysis. Crit Care Med 2013;41:1543-1554.

87 Brahmbhatt N, Murugan R, Milbrandt EB: Early mobilization improves functional outcomes in critically ill patients. Crit Care 2010;14:321.

88 Weimann A, Braga M, Harsanyi L, et al: ESPEN Guidelines on Enteral Nutrition: surgery including organ transplantation. Clin Nutr 2006;25:224-244. 